\title{
REMOTE CONTROL SYSTEM FOR A MOBILE PLATFORM WITH FOUR MECANUM WHEELS
}

\author{
Luís Sarmento ${ }^{1}$, Francisco Nunes ${ }^{2}$, Ricardo Santos Martins ${ }^{3}$ \\ João Sepúlveda ${ }^{4}$,João Sena Esteves ${ }^{5}$ \\ 1,2,3 Department of Industrial Electronics \\ University of Minho, Guimarães, Portugal \\ 4,5 R\&D Centre Algoritmi \\ University of Minho, Guimarães, Portugal \\ a66160@alunos.uminho.pt,a71213@alunos.uminho.pt,a72181@alunos.uminho.p,tmjs@dei.uminho.pt,
} sena@dei.uminho.pt

\begin{abstract}
Providing the industry with efficient transportation systems is of utmost importance and academic research on this topic deserves encouragement from governmental institutions. In many workplaces the space is reduced. Ensuring the required manoeuvrability of mobile platforms inside such environments is quite a challenge. Anthropomorphic robots are not a viable option in most situations. Usually, mobile platforms - including mobile robots - with wheels are the most cost effective option, but the use of traditional steering systems leads to severe constraints regarding freedom of movements. The use of omnidirectional steering systems allows overcoming this limitation. Since its invention, Mecanum wheels have been used on various mobile platforms, including Automatic Guided Vehicles (AGV), enabling them to perform omnidirectional movements. This paper describes a robust, intuitive and functional remote control system for a mobile platform with four Mecanum wheels, capable of performing rotation movements, translation movements and movements resulting from simultaneous rotation and translation. The system was designed in order to maximize the freedom of movements of the platform. A graphical interface for monitoring several parameters inherent to the operation of the platform is also presented.
\end{abstract}

Keywords: Omnidirectional Mobile Platform, Mecanum Wheels, Mobile Robots.

\section{Introduction}

Industries are in permanent look for more efficient transportation systems, since most of the times the space to maneuver is reduced. Mobile platforms with traditional steering systems, based on Ackerman steering geometry, are an option to take in consideration, but they have some limitations regarding the freedom of movements.

The development of steering systems granting more freedom of movements and, consequently, more efficiency, is a subject of great interest. The use of omnidirectional steering systems allows overcoming this limitation; namely, Mecanum wheels have been used on various mobile platforms, including Automatic Guided Vehicles (AGV) enabling them to perform omnidirectional movements. This paper presents a robust, intuitive and functional remote control system for a mobile platform with four Mecanum wheels, capable of performing rotation movements, translation movements and movements resulting from simultaneous rotation and translation.
The system was designed in order to maximize the freedom of movements of the platform.

In this first chapter of the paper, a distinction between an omnidirectional mobile platform and a non-omnidirectional mobile platform is made. The framework, motivations and objectives are also presented, as well as a gathering of remote control systems already implemented. In the second and third chapters, the Mecanum wheel and the steering systems with four Mecanum wheels are briefly introduced. Afterwards, from chapters 4 to 6 , the solution developed by the authors is presented. The remaining chapters present some functionality tests, conclusions and ideas for future developments.

\subsection{Omnidirectional Mobile Platform}

A vehicle that can drive with 3 Degrees of Freedom (DOF) in a bi-dimensional space is considered an omnidirectional moving platform. It is possible to control, independently, the translation and the rotation of such a mobile platform [1]. 
An omnidirectional platform has important advantages comparatively to non-omnidirectional platforms, including the following [2]: (i) Optimization of mobile platform trajectories. With the use of systems with these features, the distance that is necessary to travel from one position and orientation to another is minimized. This is due to the fact that this platform allows translational and rotational movements simultaneously.

Figure 1 [1] shows the trajectory of a nonomnidirectional vehicle when it moves from a point $A$ to a point $B$. It is possible to conclude that the distance the vehicle has to travel to reach its destination is more than two times the distance in straight line between point $\mathrm{A}$ and point $\mathrm{B}$.

Figure 2 [1] shows the trajectory described by an omnidirectional vehicle between the same points $A$ and B; (ii) Possible reduction of energy consumption. Minimizing the distance travelled by the platform may result in a reduction of energy consumption.

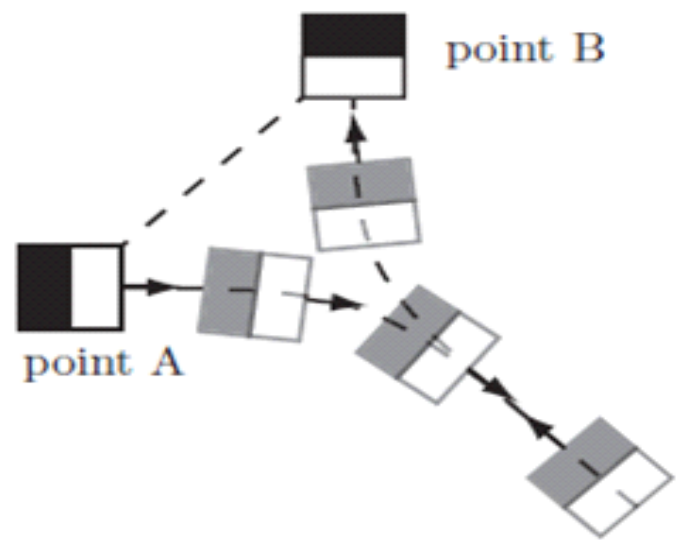

Figure 1: Path described by a non-omnidirectional vehicle to go from point $A$ to point $B$ [1].

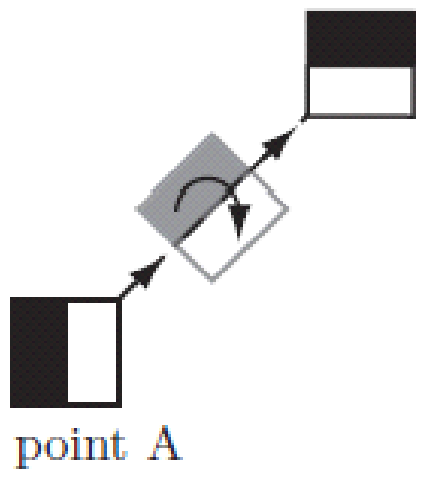

point B

Figure 2: Trajectory described by an omnidirectional vehicle to go from point $A$ to point $B$ [1].

\subsection{Framework}

In many applications, the use of non-omnidirectional steering systems on mobile platforms (particularly mobile robots) is a severe limitation in terms of freedom of movement. In fact, platforms with a traditional steering system (non-omnidirectional platforms), navigating in a xOy plane, do not allow movements with more than 2 DOF [1].

Most of the Automatic Guided Vehicles (AGV) used in industry have 2 DOF, which limits the number of possible movements. This restriction lead to a difficulty, or even impossibility, to perform certain tasks in places where the space for navigation is reduced [3].

\subsection{Motivation}

Taking into account the problem presented in the previous subchapter, a possible solution to the above mentioned limitations is the use of an omnidirectional platform with 3 DOF based on four Mecanum wheels. Even though it is rare in everyday life, this configuration is used in some specific applications, such as (i) in vehicles to explore the Moon and Mars in possible future missions, (ii) forklift trucks, (iii) omnidirectional wheelchairs, among some others [2]. Figure 3[2] shows some of these applications.

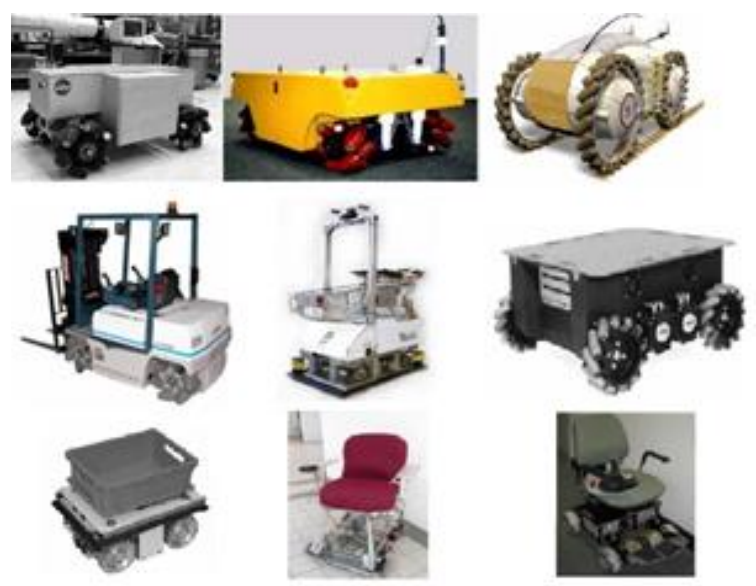

Figure 3: Examples of application for the steering system based on four Mecanum wheels [2].

\subsection{Objectives}

The main objective of the work is the development of a remote control system for a mobile platform with four Mecanum wheels. This remote control system must allow control over the 3 DOF of the steering system of the platform. 
The system requirements are the following: (i) The remote control should be robust, easy to use and intuitive; (ii) The remote control should maximize both rotational and translational movements of the 3 DOF mobile platform.

\subsection{State of the art}

Most of the solutions regarding the control of omnidirectional platforms use joysticks, which are used since the beginning of computer games. The most common joysticks use potentiometers working as a sensors to output analogue voltages. Figure 4 [4] shows a block diagram of a remote control with one joystick responsible for the translational movements combined with two buttons, which are responsible for the rotational movements [4].

Another simple approach is to have a second joystick instead of the two buttons. In this second joystick only the $\mathrm{X}$ axis is used, to rotate the platform clockwise or counter-clockwise. This is a very intuitive solution. Many gaming controllers and model car remote controllers have two joysticks.

Since gaming controllers were mentioned, another approach has to be reminded. A gaming controller can be connected to a microcontroller in order to remotely control the mobile platform. Figure 5 [5] shows an example of this kind of solution. It is almost the same solution described in the last paragraph. Besides being an intuitive solution, ergonomics is also guaranteed.

Apart from the use of joysticks, there are other possible solutions. A wheeled based system combined with two pedals may be used to drive the platform. This solution is also intuitive for almost all the users. Figure 6 [6] shows an example of this approach.

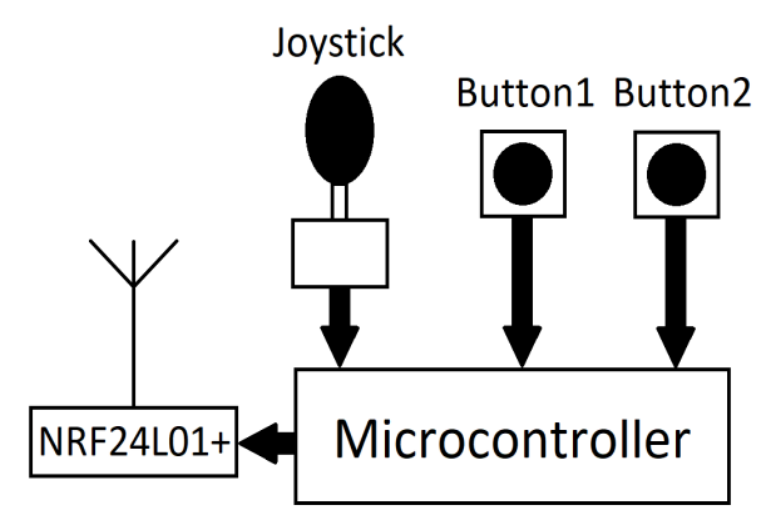

Figure 4: Block diagram of a remote control with one joystick and two buttons [4].

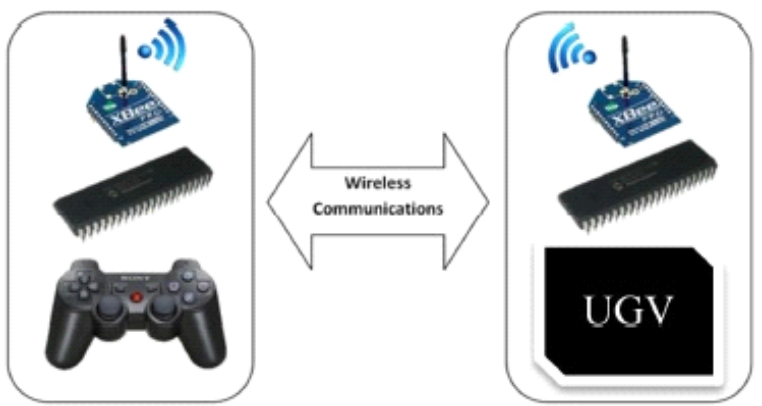

Figure 5: Example of a remote control responsible for driving an UGV [5].

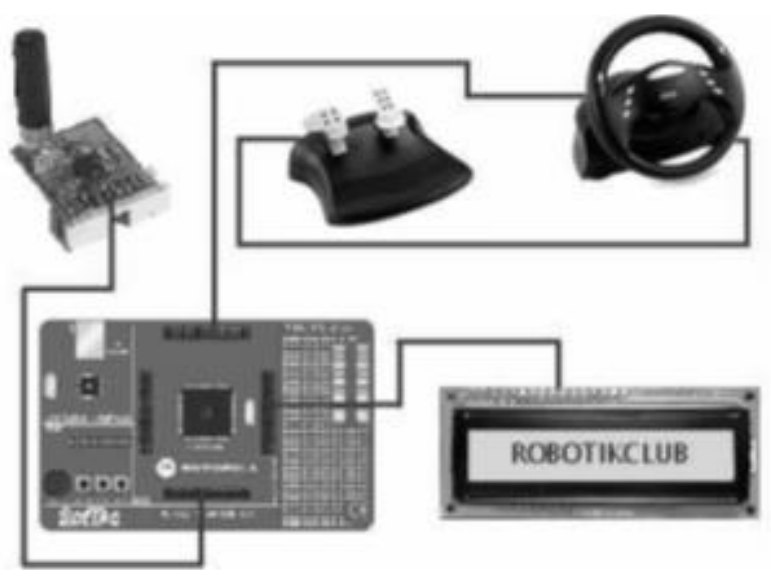

Figure 6: Wheeled based system used to control an omnidirectional mobile platform [6].

The last solution presented in this subchapter uses a Penny+Gilles JC2000 Fingertip 3 axis industrial joystick [7], shown in Figure 7 [7]. Instead of potentiometers, this joystick use three Hall Effect sensors to output analog signals ranging from $0.5 \mathrm{~V}$ to $4.95 \mathrm{~V}$.

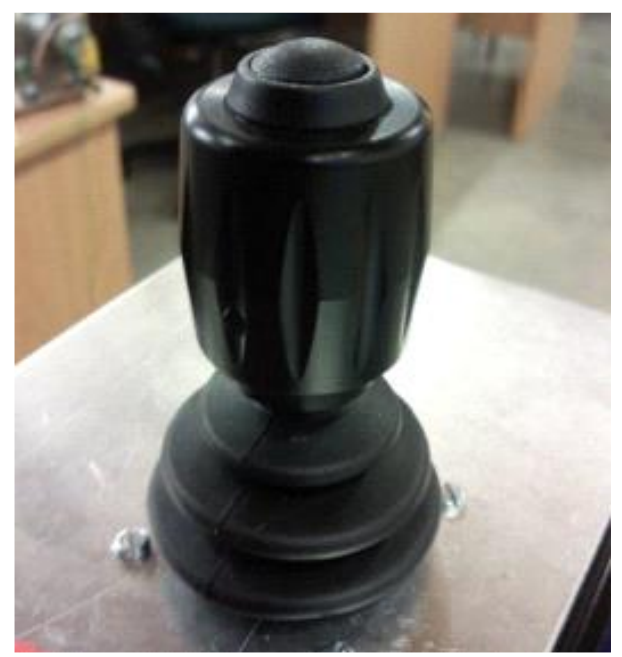

Figure 7: Penny+Gilles JC2000 Fingertip 3 axis industrial joystick [7]. 


\section{The Mecanum Wheel}

The Mecanum Wheel (Figure 8 [7]), invented in 1973 , consists of a set of rolls symmetrically placed around the wheel body. In the case of the conventional Swedish wheel, the angle the axis of each roll makes with the rotation axis of the wheel is 45으. [8]. By using four Mecanum wheels, it is possible to build an omnidirectional mobile platform.

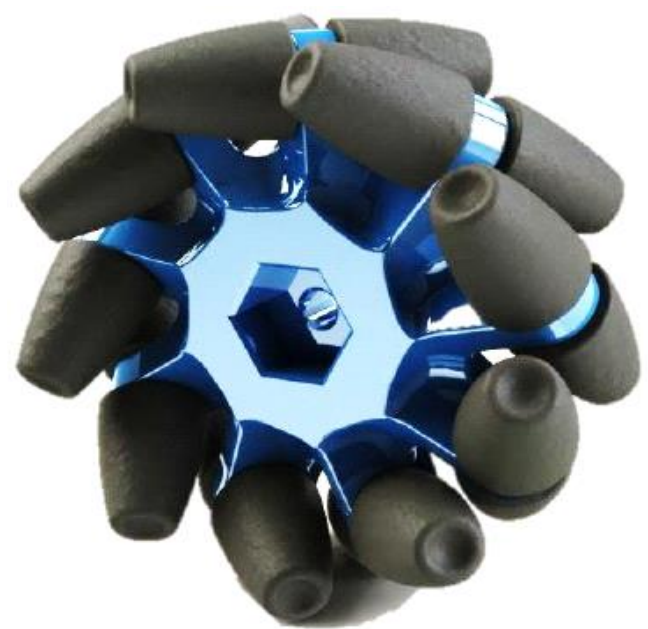

Figure 8: Design and basic components of a Mecanum wheel [7].

The main advantages of Mecanum wheels are (i) the ability to carry heavy loads (especially if the rollers are attached at the ends), (ii) the existence of 3 DOF in mobile platforms with this kind of wheels and (iii) the high traction capacity when compared to omnidirectional wheels (Figure 9 [7]). However, there are also some drawbacks when using this type of wheels such as (i) discontinuous wheel contact with the ground, (ii) high sensitivity to the irregularities of the ground, (iii) complex mechanical design and (iv) high price (when compared to the conventional wheels) [9].
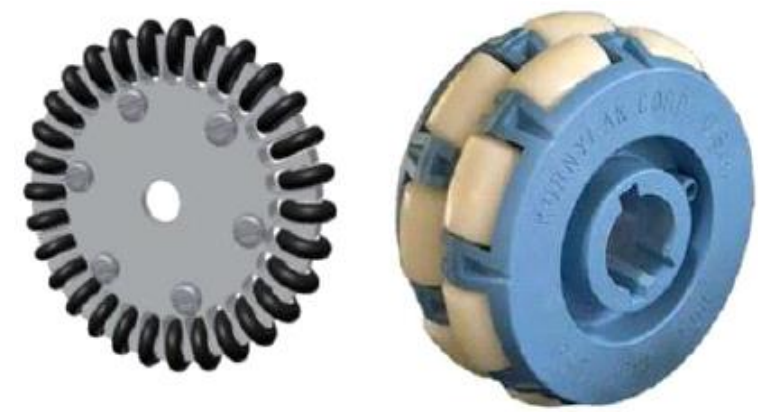

Figure 9: Two designs of omnidirectional wheels [7].

\section{Steering Systems with four Mecanum Wheels}

Four-wheeled steering systems are very common. They are used, for example, in cars. Usually, they are able to carry heavier loads than any other steering systems with less than four wheels. The arrangement of the wheels usually forms a square or a rectangle. To maximize stability, the mass centre of the mobile platform must match the geometric centre of these figures [10] [11].

The mobile platform used in this project (Figure 10) is omnidirectional, with four Mecanum wheels. Its steering system (Figure 11 [2]) is governed by the following mathematical model [7] [12] [13]:

$$
\left[\begin{array}{l}
V 1 \\
V 2 \\
V 3 \\
V 4
\end{array}\right]=\left[\begin{array}{ccc}
1 & 1 & -\frac{C+L}{2} \\
-1 & 1 & \frac{C+L}{2} \\
-1 & 1 & -\frac{C+L}{2} \\
1 & 1 & \frac{C+L}{2}
\end{array}\right] *\left[\begin{array}{c}
V x \\
V y \\
W z
\end{array}\right]
$$

Where:

- $\mathrm{Vx}$ is the linear velocity of the mobile platform along the $\mathrm{X}$ axis;

- Vy is the linear velocity of the mobile platform along the $\mathrm{Y}$ axis;

- $\mathrm{Wz}$ is the angular velocity of the mobile platform;

- $\mathrm{L}$ is the distance between the centres of wheels 1 and 2 or wheels 3 and 4 (figure 11);

- $\mathrm{C}$ is the distance between the centres of wheels 1 and 3 or wheels 2 and 4 (figure 11);

- V1 is the linear velocity of wheel 1 ;

- V2 is the linear velocity of wheel 2;

- V3 is the linear velocity of wheel 3;

- V4 is the linear velocity of wheel 4.

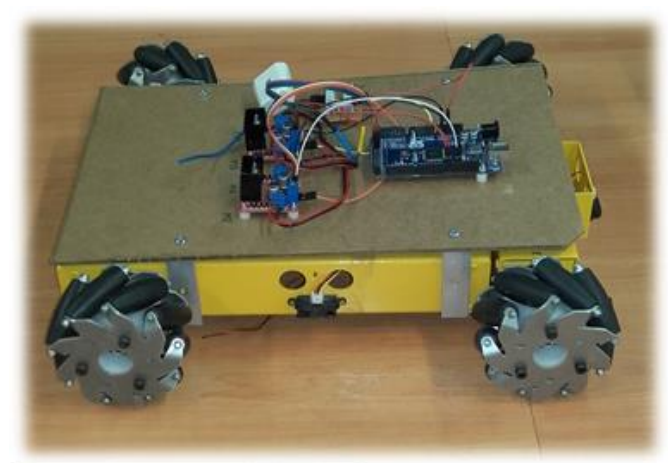

Figure 10: Omnidirectional mobile platform with four Mecanum wheels, used in this project. 


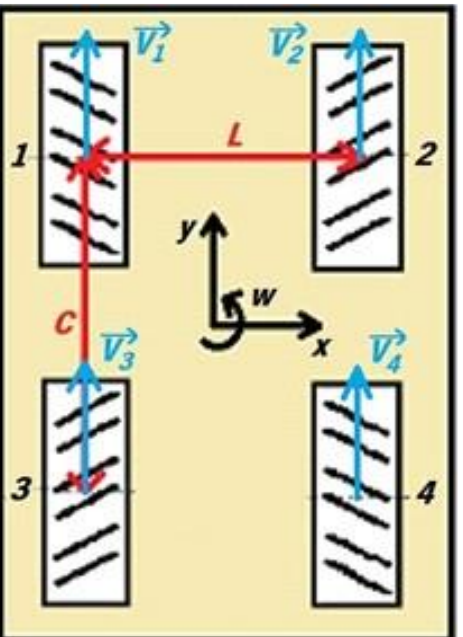

Figure 11: Omnidirectional steering system with four Mecanum wheels [2].

This mathematical model makes it possible to compute the linear velocity to be applied to each of the four Mecanum wheels, in order to make any desired movement in a plane. It is important to note that the mathematical model referred is valid only when all the wheels are in contact with the ground.

The conversion between linear velocity and angular velocity is given by the following equation:

$$
v=W * T
$$

Figure 12 [4] shows all the basic movements for this configuration - there are intuitive movements (for example, frontal movement) and movements whose direction of rotation of the wheels is not obvious (for example, diagonal movements). In figure 12 [4], for each situation, the wheels that are not marked with an arrow have a zero speed.

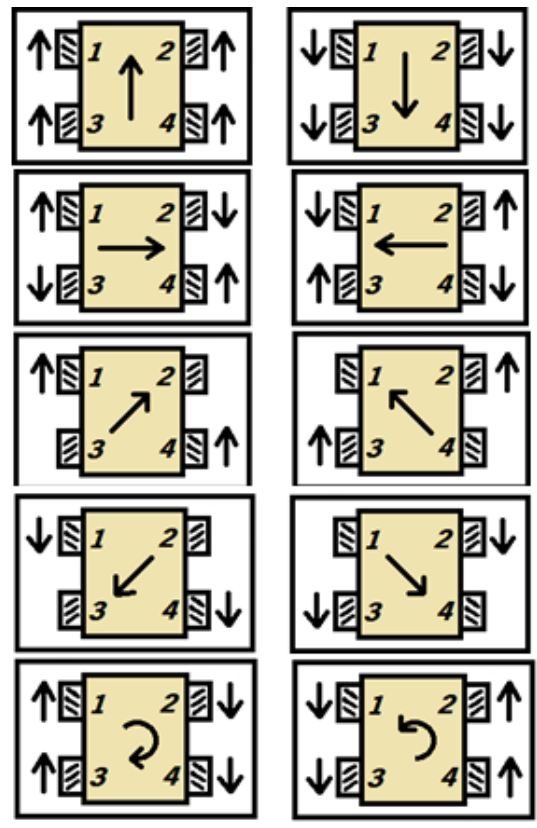

Figure 12: Possible movements of an omnidirectional mobile platform with four Mecanum wheels [4].

\section{Proposed Solution}

In this work, the proposed solution for controlling the mobile platform is based on a JH-D400X-R2 three axis joystick with three potentiometers (Figure 13). The first two axis are associated to the translational movement of the platform. The third axis is associated to the rotational movement of the platform. There is also a push button, which will be important to change the driver's orientation (the concept will be clarified in the next chapter). An Arduino Uno connected to the joystick is responsible to receive data from the joystick and decode it. Then, this data is sent to an Arduino Mega placed in the car, in order to actuate the motors.

With a single device, it is possible to control a wide variety of platform movements in a very intuitive way. Also, it is possible to drive the vehicle at different velocities. All things considered, this is a cost-effective, intuitive, compact and robust solution. It may not be as precise as the Penny+Gilles JC2000 Fingertip 3 axis industrial joystick previously mentioned, but the precision achieved is enough for its purpose, and the cost is significantly lower.

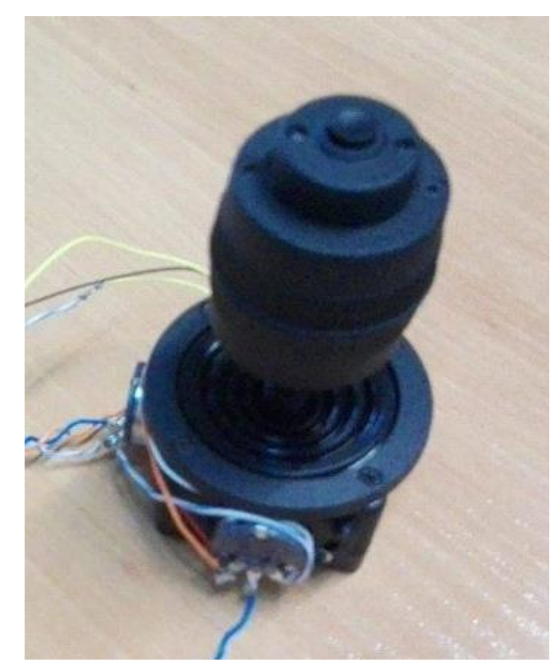

Figure 13: Joystick used to control the mobile platform.

\section{Navigation}

An interesting approach may be applied to the navigation control, taking advantage from the fact that the platform allows a great variety of movements. Since the driver is not on the platform (it is remotely controlled), it is more intuitive that the front heading direction of the vehicle is dictated by the human front direction. This means that, regardless of the heading of the vehicle, if the driver moves the joystick forward, the vehicle will move forward too (even if it is facing left, right or behind). This navigation technique may be found in high-end drones, where it is called "headless mode". 
However, as far as the authors are aware, it is a novelty when applied to mobile platforms with Mecanum wheels.

The described navigation technique implies determining the heading direction of both the driver and the vehicle. The vehicle was equipped with a 3 DOF IMU (MPU 6050), used in order to determine its heading direction. Clicking the joystick button sets the same heading direction for both the driver and the vehicle.

Regarding the motion maths, the transformation of Cartesian coordinates to polar coordinates proved to be very useful. However, the information read from the joystick and applied to the motors are in Cartesian form, so the transformation and its inverse have to be continuously performed. Equation 3 is used to convert the data from the joystick to polar coordinates.

$$
\left\{\begin{array}{l}
r=\sqrt{x^{2}+y^{2}} \\
\theta_{d}=\tan ^{-1}\left(\frac{y}{x}\right)
\end{array}\right.
$$

On the above equation, $r$ represents the linear velocity and $\theta_{d}$ is the desired heading direction.

Assuming that there is a variable "angle" that stores the current heading direction of the vehicle and "d_angle" is the driver heading direction, equation 4 determines the final heading direction $\theta_{f}$ the vehicle should move.

$$
\theta_{f}=\text { angle }-d_{\text {angle }}-\theta_{d}
$$

With the velocity ${ }_{r}$ (computed from equation 3 ) and the final heading direction $\theta_{f}$ (computed from equation 4), it is necessary to reconvert these coordinates to Cartesian ones (equation 5), applicable to the vehicle.

$$
\left\{\begin{array}{l}
v_{x}=r \cos \left(\theta_{f}\right) \\
v_{Y}=r \sin \left(\theta_{f}\right)
\end{array}\right.
$$

On the above equations, $\mathrm{x}$ and $\mathrm{y}$ are the data read from joystick; $r$ and $\theta_{f}$ are the final polar coordinates; and $v_{x}$ and $v_{y}$ are the linear velocities to be applied to the matrix (equation 1).

Considering that this project has two main components (the remote controller and the vehicle), it is important to distinguish their roles, as well as the communication between them. In order to do so, the flowchart shown in Figure 14 represents the main control loop of the remote controller. The flowchart shown in Figure 15 represents the main control loop of the vehicle.

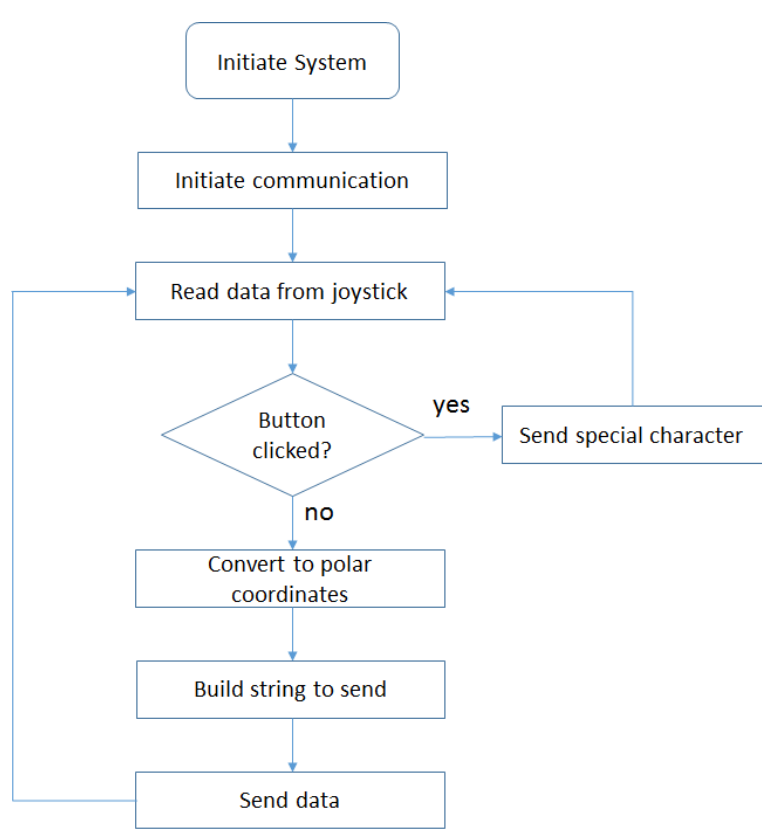

Figure 14: Flowchart of the main control loop of the remote controller

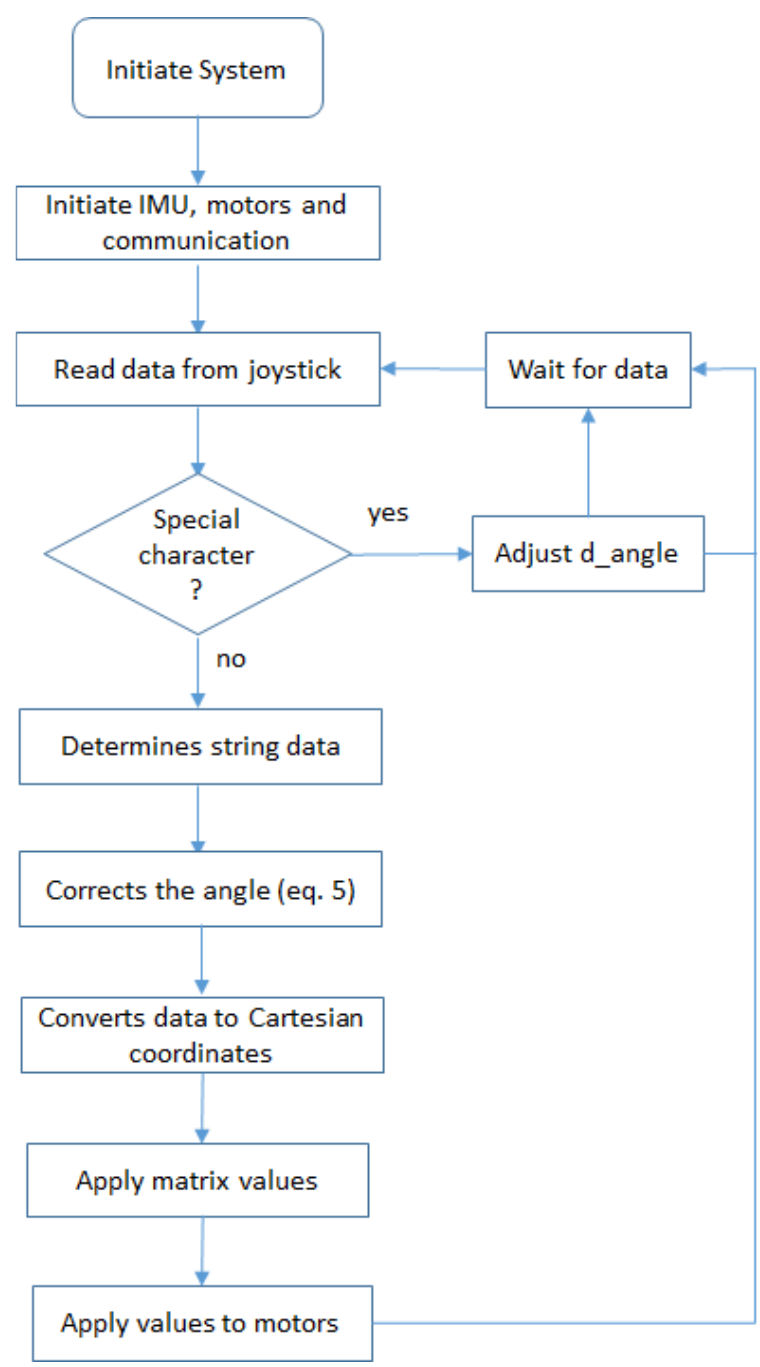

Figure 15: Flowchart of the main control loop of the vehicle. 


\section{Wireless Communication.}

Since one of the work requirements was to develop a remote control provided with wireless communication, several known options were studied.

The authors decided to choose XBee, because it can completely fulfil the requirements of the work. In addition, it can achieve data transfer rates up to $250 \mathrm{kbps}$, which is enough for the purpose of the work. Also, it has a relatively good range of distance (it can go up to $1500 \mathrm{~m}$, in line of sight), which is an advantage to take in consideration. The low-power consumption was an important factor taken into account, too.

Using XBee, different network topologies can be created. In this particular case, point-to-point communication seemed to be the better option. Therefore, an XBee module was used on the mobile platform working as a coordinator and another placed in the remote control working as a router.

\section{Functionality Tests}

With the control algorithms represented in Figure 14 and Figure 15 implemented and installed in the vehicle and the remote controller, the new navigation approach was tested successfully. The vehicle navigates as expected, according to the joystick movements, the way described in Chapter 5 . The heading direction of the vehicle is updated each time the joystick button is pressed.

The voltage at each motor was measured while three predefined movements were performed in the joystick. Firstly, the joystick was pushed forward to the maximum amplitude. All the motors were supposed to rotate with the same speed and in the same direction, which requires equal voltages applied to them. The voltages measured at the motors are presented in Table 1.

Afterwards, the vehicle was rotated clockwise by twisting the joystick. Finally, the joystick was pushed forward again to the maximum amplitude. Now, motors 2 and 3 should have the same voltage shown in Table 1 . Motors 1 and 4 should have voltages with the same modulus as the voltages shown in Table 1, but negative.

Table 2 shows the voltages measured at the motors while the joystick is pushed forward, after the rotation of the platform.

Table 1: Voltages measured at the motors while the joystick is pushed forward.

\begin{tabular}{|c|c|c|c|}
\hline MOTOR 1 & MOTOR 2 & MOTOR 3 & MOTOR 4 \\
\hline $10.41 \mathrm{~V}$ & $10.33 \mathrm{~V}$ & $10.45 \mathrm{~V}$ & $10.49 \mathrm{~V}$ \\
\hline
\end{tabular}

Table 2: Voltages measured at the motors while the joystick is pushed forward, after a rotation of the

platform.

\begin{tabular}{|c|c|c|c|}
\hline MOTOR 1 & MOTOR 2 & MOTOR 3 & MOTOR 4 \\
\hline$-10.31 \mathrm{~V}$ & $10.40 \mathrm{~V}$ & $10.46 \mathrm{~V}$ & $-10.39 \mathrm{~V}$ \\
\hline
\end{tabular}

A video demonstration is available at the following website: https://youtu.be/5jyH4U5PlTk

\section{Conclusions and Future Work}

It is a fact that mobile platforms based on Mecanum wheels can perform much more movements than traditional vehicles. It is also a fact that there are several applications for this type of platforms, both in the military and industrial fields. They are of the most interest both for governments and industries. Taking that into consideration, the relevance of the presented work arises: an intuitive and yet robust remote control for an omnidirectional platform with four Mecanum wheels. The system presented in this paper makes it possible, with a single device, to control all the movements of that mobile platform. In addition, the user does not need to concern about which direction the front of the vehicle is heading. These approaches make the solution presented advantageous regarding other remote controls with several buttons and joysticks.

Regarding future work, an autonomous navigation mode is suggested. Since omnidirectional mobile platforms may be used in industrial environments to perform repetitive tasks, such a feature would be a great advantage. The development of a graphical user interface would improve the interaction between the mobile platform and the user.

\section{Acknowledgements}

The authors would like to express their acknowledgments to COMPETE: POCI-01-0145FEDER-007043 and FCT - Fundação para a Ciência e Tecnologia within the Project Scope: UID/CEC/00319/2013.

\section{References}

[1] R.P.A. van Haendel, "Design of and omnidirectional universal mobile platform", DCT 2005.117, DCT traineeship, Eindhoven, September 2005.

[2] Carlos Alberto Fernandes Arantes, "Desenvolvimento de uma plataforma móvel omnidirecional baseada em rodas Mecanum com gestão otimizada da energia"; master thesis for the Integrated Master on Industrial, Electronics 
and Computers Engeneering, University of Minho, December 2014.

[3] Florentina Adãscãlitei, Ioan Doroftei, "Practical Applications for Mobile Robots based on Mecanum Wheel - a Systematic Survey", Gh.Asachi Technical University of Iasi, Mechanical Engineering Faculty, Theory of Mechanisms and Robotics Department, B-dul D. Mangeron, 63-63, 70050, Iasi, Romania, MECAHITECH'11, Vol. 3, 2011.

[4] M. K. S. H. Maldeniya, R. C. Madurawe, L. B. H. T. Thilakasiri, T. M. S. Thennakoon, R. M. T. P. Rajakaruna, "Remote Controlled 4WD Omni Directional Robot Using Mecanum Wheels"; Department of Mechatronics, Faculty of Engineering, South Asian Institute of Technology and Medicine (SAITM), Sri Lanka, 2015

[5] M. Z. H. Noor, S. A. S. M. Zain, L. Mazalan, "Design and Development of Remote-Operated MultiDirection Unnamed Ground Vehicle (UGV)", Faculty of Electrical Engineering, University Teknologi Mara, Shah Alam, Malaysia, August 2013

[6] I. Doroftei, V. Grosu, V. Spinu, "Omnidirectional mobile robot - design and implementation" in Bioinspiration and Robotics: Walking and Climbing Robots, Austria, Vienna:I-Tech, pp. 544, Sep. 2007

[7] Ryan Thomas, "Omni-Directional Mobile Platform for The Transportation of Heavy Objects", Massey University, Palmerston North, New Zealand, 2011.
[8] Bengt Ilon, "Wheels for a course stable selfpropelling vehicle movable in any desired direction on the ground or some other base", US Patent 3876255 A, April 8, 1975.

[9] Stephen L. Dickerson, Brett D. Lapin, "Control of an Omnidirectional Robot Vehicle with Mecanum Wheels", Georgia Institute of Technology, Conference, 1991. Proceedings. Vol. 1, NTC '91, National.

[10] Roland Siegwart, Illah R. Nourbakhsh, Davide Scaramuzza, "Introduction to Autonomous Mobile Robots", Second Edition, ISBN 978-0262-01535-6, Massachusetts Institute of Technology, Massachussetts, USA, 2011.

[11] Kristof Goris, "Autonomous Mobile Robot Mechanical Design”, Free University of Brussels, 2005.

[12] Jungmin Kim, Jungje Park, Sungshin Kim, "Inertial Navigation System for OmniDirectional AGV with Mecanum Wheel", School of Electrical Engineering, Pusan National University, Geumjeong, Busan 609-735, Korea, Advances in Mechanical Engineering, ISSN: 2160-0619, Vol.2, No. 1, March 2012.

[13] Christof Rohrig, Daniel Heb, Christopher Kirsch, Frank Kunemund, "Location of an Omnidirectional Transport Robot Using IEEE 802.15.4a Ranging and Laser Range Finder", The 2010 IEEE/RSJ International Conference on Intelligent Robots and Systems, Taipei, Taiwan, October 18-22, 2010. 